\title{
Modelling Unidirectional Crowd Motion in a Corridor with Statistical Characteristics of Pedestrian Movements
}

\author{
Tan Chen, ${ }^{1,2,3}$ Wei Wang $\mathbb{D}^{1,2,3}$ Yu Tu, ${ }^{1,2,3}$ and Xuedong Hua $\mathbb{D}^{1,2,3}$ \\ ${ }^{1}$ Jiangsu Key Laboratory of Urban ITS, Southeast University, Nanjing 210096, China \\ ${ }^{2}$ Jiangsu Province Collaborative Innovation Centre of Modern Urban Traffic Technologies, \\ Southeast University, Nanjing 210096, China \\ ${ }^{3}$ School of Transportation, Southeast University, Nanjing 210096, China \\ Correspondence should be addressed to Wei Wang; wangwei_transtar@163.com
}

Received 8 October 2019; Accepted 27 May 2020; Published 30 June 2020

Academic Editor: Sergey A. Suslov

Copyright (c) 2020 Tan Chen et al. This is an open access article distributed under the Creative Commons Attribution License, which permits unrestricted use, distribution, and reproduction in any medium, provided the original work is properly cited.

\begin{abstract}
Lattice gas model is a kind of mature and convenient pedestrian simulation model. The original lattice gas model adopts discontinuous step length and finite moving directions to simulate crowd motion, which will lead to some unreasonable movements; besides, the transition probability used in this model is often manually designed and lacks the verification of realistic pedestrian trajectories. Based on an open pedestrian trajectory dataset, we first derived the relationship between local density and the distribution of pedestrian movements' length and then proposed an extended lattice gas model considering the statistical characteristics of pedestrian movements, which extends the concept of transition probability in the original lattice gas model to distribution of pedestrian movements' length in two perpendicular directions. The proposed model is applied to a scenario which is the same as the experiments of the open dataset, and the numerical results demonstrate that the proposed model can reproduce the fundamental diagrams and the transition probability of the experimental dataset well. This study is helpful to understand the statistical characteristics of pedestrian movements and can improve the applicability and accuracy of the lattice gas model.
\end{abstract}

\section{Introduction}

The research studies on pedestrian behaviours can benefit our perception of the crowd dynamic evolution and improve the design of pedestrian facilities inside the building and on the street, such as exits in the room $[1,2]$, crosswalks at the intersection $[3,4]$, and aisles in the metro station $[5,6]$. Traditional research studies on pedestrian dynamic mainly focus on corridors [7-9] and exits [7, 10, 11], as they are the most common crowd motion scenarios, yet recent research studies also extended to numerous situations, such as stairs [12], corner [13], and multiple-angle crossing [14, 15]. Besides, many interesting or critical factors that may have an effect on crowd motion, for instance, background music [16], visibility [17], disabled people [18, 19], and artificial attack [20], are also be investigated. Some research studies use experimental data collected by cameras to understand the pedestrian behaviours $[13,15-17,21-25]$, while others adopt microscopic simulation models to reproduce the movements of the crowd $[1,7,8,26-28]$. The existing microscopic pedestrian flow simulation models can be divided into two categories, force-based models and rule-based models.

Force-based models use the force concept in Newtonian mechanics to explain the behaviour of a moving pedestrian, such as social force model [7] and centrifugal force model [26]. These force-based models can denote the interaction between a pedestrian and other traffic participants and are successfully applied to mimic the pedestrian movements in numerous situation, e.g., crosswalk, canteen, and deck $[3,4,29,30]$, but force-based models require high computational power to determine pedestrian position. Meanwhile, some psychological parameters used in these models are relatively difficult to calibrate. Rule-based models use particles to represent pedestrians and set several rules to guide particles to move in a system. The most typical rule-based model is the cellular automaton model. Blue and Alder used the cellular automation model with a small rule 
set to simulate pedestrian flow in several conditions and reproduced the microscopic pedestrian movements and macroscopic activities [9, 27, 31]. Then, numerous studies improved Blue and Alder's model to make it suitable for simulating herding effect [10], group behaviour [2], clogging [32], etc. The lattice gas model (LGM) is a popular modified version of the cellular automata model. Due to its convenience in programming and high efficiency, LGM has attracted extensive research studies after it was proposed by Muramatsu in 1999 [8]. Recently, some LGMs have tried to redefine the shape of the grid to obtain better pedestrian simulation results. To study pedestrian dynamic under a high density, Ji proposed a triangular grid model with an area of $0.125 \mathrm{~m}^{2}$ and 14 potential next step positions [33]. Qiu et al. applied square and triangular LGM on $Y$-shaped channel and studied the relationship between merging flow density and channel width and angle [6]. Others try to incorporate more factors to LGM. Tu and Zhou introduced memory effect to LGM for simulate bidirectional pedestrian flow and found that length of pedestrian's memory can cause instability and jam to the flow [34]. Miyagawa and Ichinose assumed an evacuee will occupy 2 adjacent grids and the pedestrian's turning behaviour can be described [35]. Ding et al. studied the transition probabilities under intersecting pedestrian flow on the $2 \mathrm{D}$ lattice with the parallel update rule [36].

As shown in Figure 1, the original LGM uses biased random walkers to mimic pedestrian behaviour and a pedestrian will move to the left, right, or forward lattice in the next step, or keep stationary, by a given transition probability. The probability depends on the strength of drift $(D)$, which is usually a giving value between $[0,1]$. There are eight configurations of a particle and the transition probability is $P_{x}=D+(1-D) / 3, \quad P_{y}=(1-D) / 3$, and $P_{-y}=(1-D) / 3$ for configuration (a), $P_{x}=D+(1-D) / 2$ and $P_{y}=(1-D) / 2$ for configuration (b), $P_{x}=D+(1-D) / 2$ and $P_{-y}=(1-D) / 2$ for configuration (c), $P_{y}=1 / 2$ and $P_{-y}=1 / 2$ for configuration (d), $P_{x}=1$ for configuration (e), $P_{y}=1$ for configuration (f), $P_{y}=1$ for configuration (g), and $P_{x}=P_{y}=P_{-y}=0$ for configuration (h).

However, the original LGM is somewhat different from the real pedestrian movements in some aspects. Figure 2 illustrates a right walking pedestrian and the potential positions that he/she might move to in the next step. From the comparison between Figures 1 and 2, it can be found that, firstly, a pedestrian can only move in 3 fixed directions in the original LGM, namely, right, left, and forward. However, in reality, pedestrian's directions are usually not straight, they often move in a skewed direction. Some recent studies have extended the LGM to 8 or more directions and thus have a better simulation result, yet in these models pedestrian's directions are still limited in a finite set $[1,37,38]$. Secondly, most of the previous LGMs set the length of pedestrian step to a fixed value, e.g., $0.4 \mathrm{~m}$, which neglects the diversity of pedestrian step size. This will also lead to some unreasonable movements, such as that pedestrians would prefer to stay stationary when they would collide with others, but in practice they will choose a shorter step [5]. Finer grids or more discrete pedestrian step size can improve the simulation result while these models still lead to the discontinuous pedestrian movements [5, 28, 32]. Furthermore, in many previous LGMs, the transition probability to each direction and different distances is always manually designed, which lacks the verification of realistic pedestrian trajectories [39]. For a scenario shown in Figure 3, we discretize the space into many $0.4 \times 0.4 \mathrm{~m}^{2}$ grids. It can be seen that most pedestrian's positions are always disordered, namely, they are not keep in align as shown in the original LGM. Two right walking pedestrians who face with different levels of crowding are shown in the left part of the figure, with their positions of the next step marked in orange. The upper one's surrounding area is more crowded than the lower, so the upper one will have a shorter step length. Besides, it can be seen that askew moving directions can be more realistic. So, the direct use of the original LGM will bring some limitations to pedestrian dynamics.

In this study, firstly, we divided the two-dimensional pedestrian movements into two perpendicular directions, one is $X$ direction which indicates the pedestrian movements in forward or backward and the other is $Y$ direction which describes movements in left or right. Next, we introduced the concept of local density, which can successfully describe the spatial occupancy of a small area around a pedestrian, and based on this we derived the relationship between local density and pedestrian movements. An open dataset that contains a series of unidirectional pedestrian flow experiments is used here for supporting our analysis [40]. The fitting result shows that there are some statistical rules between pedestrian movements and local density, and then we proposed an extended LGM for simulating pedestrian movements using statistical methods. The model incorporates these statistical rules, and the transition probability in LGM is extended from a giving drift strength $D$ to probability density functions (PDF) of two-directional movements (Figure 4). The fundamental diagrams show that the proposed model can fit the numerical result of the original data well, and the transition probability figures imply that the model can reproduce the continuous step, unlimited heading direction, and reasonable transition probability.

The rest of the paper is arranged as follows. Section 2 introduces the experimental setup of the open dataset and then derives the distributions of pedestrian movements in two perpendicular dimensions. Section 3 describes the extended lattice gas model and Section 4 shows the simulation results of pedestrian flow. In the Section 5, we conclude the paper with a summary and a proposal for the next work.

\section{Analysis of Pedestrian Movements' Statistical Characteristics}

2.1. Data Source and Experimental Setup. The open dataset is collected from experiments carried in Düsseldorf, Germany $[15,23,40]$. These experiments are part of project BaSiGo (Bausteine für die Sicherheit von Großveranstaltungen). There were 1986 participants who joined BaSiGo and most of them were college students. Since our focus is the unidirectional pedestrian movements in a corridor, the data 


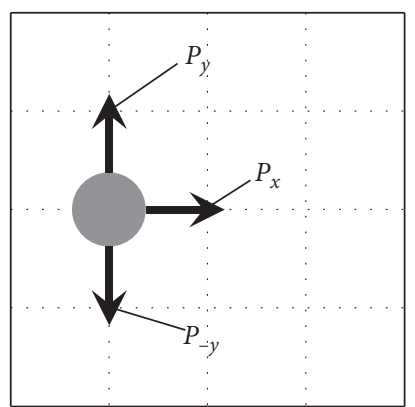

(a)

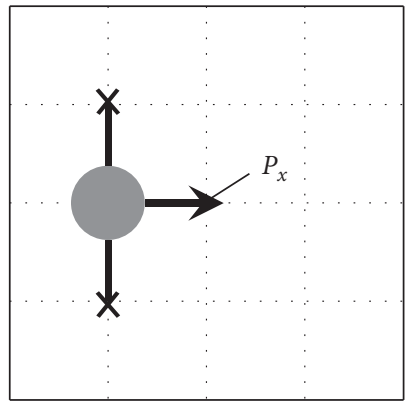

(e)

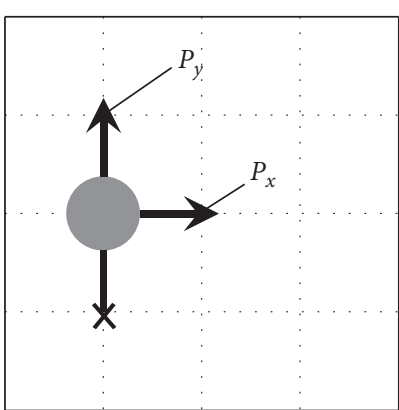

(b)

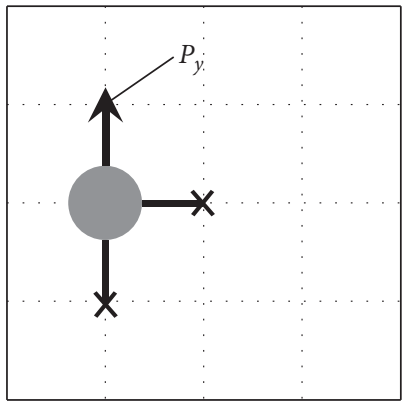

(f)

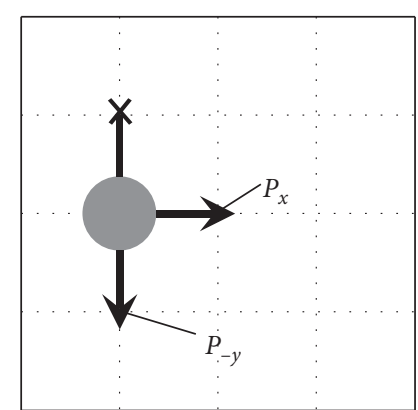

(c)

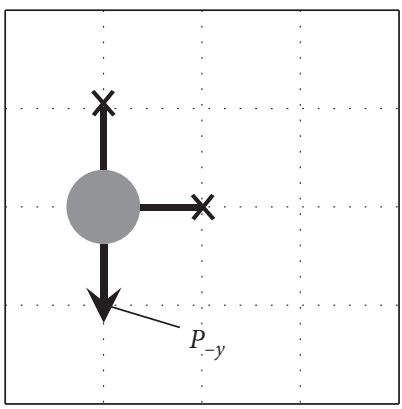

(g)

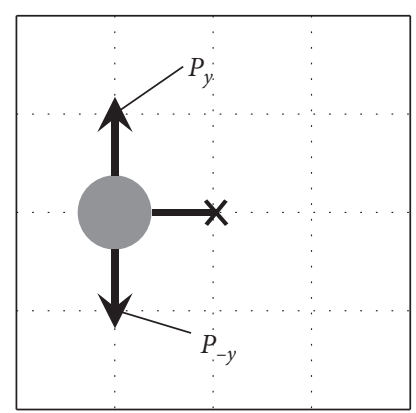

(d)

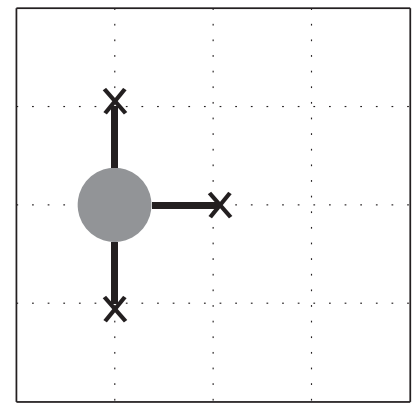

(h)

FIgUre 1: Configurations of the original LGM.

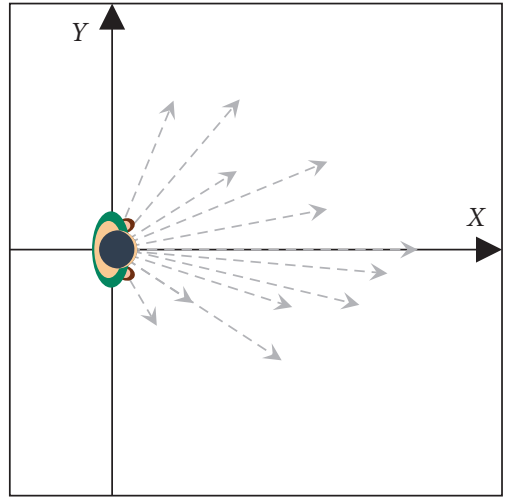

Figure 2: Pedestrian's potential movements.

extracted from unidirectional flow experiment (BaSiGo_UO) were analysed.

Figure 5 shows the layout of pedestrian flow experiments. In the experiments, pedestrians are gathering in the waiting area at the beginning of experiment, and then they enter the $16 \mathrm{~m}$ long and $W_{\text {cor }}=5 \mathrm{~m}$ wide corridor. After a pedestrian traverses the corridor, he/she is instructed to walk outside the experiment area and return to the waiting area for the next run. The width of doors in the entrance and exit of the corridor is $W_{\text {in }}$ and $W_{\text {out }}$, respectively. In each run, the global density of pedestrians in the corridor can be controlled by changing the width of these two doors. There are six runs in the experiment and the main differences among these runs are the number of participants $(N)$ and the width of doors. The tag and some geometric information of each run are shown in Table 1. Cameras on the top of the experiment areas capture the motion of crowd, and pedestrians' trajectories are

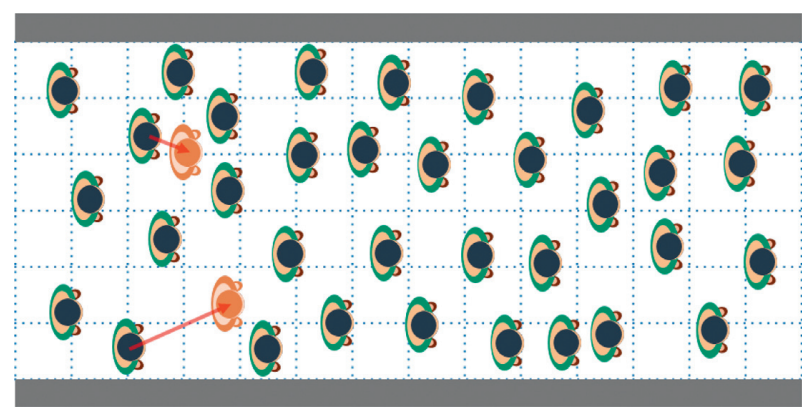

FIgURE 3: Typical simulation scenario.

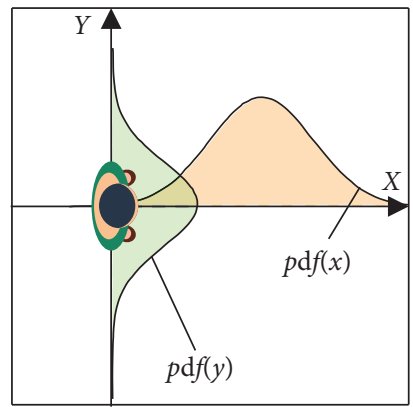

FIgURE 4: Extended lattice gas model.

extracted from these videos. The pedestrians' trajectories within the blue square, which is located in the most central part of the corridor, were brought out for studying. The length and width of this square are both $5 \mathrm{~m}$. Taking into account the computational efficiency and the accuracy of the model, we set the time step as $0.5 \mathrm{~s}$ in the following parts. 


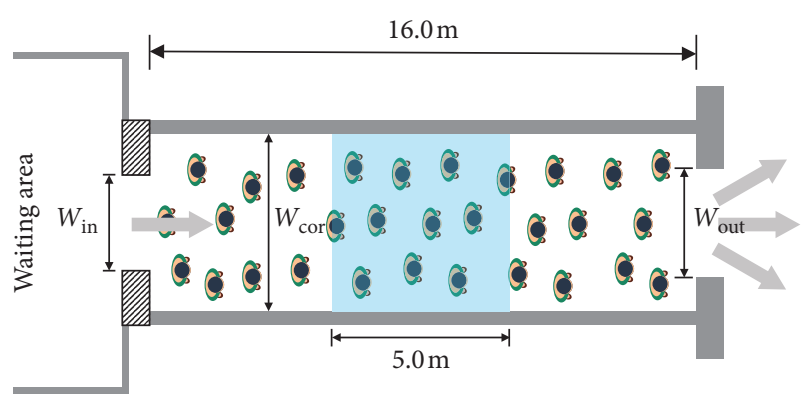

FIGURE 5: Layout of pedestrian flow experiment.

TABle 1: Tags and geometric information of experiments.

\begin{tabular}{lcccc}
\hline BaSiGo_UO & Tag & $W_{\text {in }}(\mathrm{m})$ & $W_{\text {out }}(\mathrm{m})$ & $N$ \\
\hline \multirow{4}{*}{ Unidirectional } & uni_corr_500_1 & 1 & 5 & 148 \\
flow & uni_corr_500_3 & 3 & 5 & 916 \\
& uni_corr_500_4 & 4 & 5 & 909 \\
& uni_corr_500_5 & 5 & 5 & 905 \\
& uni_corr_500_6 & 5 & 4 & 913 \\
& uni_corr_500_7 & 5 & 3 & 914 \\
\hline
\end{tabular}

2.2. Local Density Calculation Method. When a pedestrian is passing through a corridor or evacuating from a room, his/ her movement is usually influenced by some nearby pedestrians rather than the whole crowd, and it is obvious that the closer pedestrian will have a bigger influence on the subject. Therefore, the concept of local density is introduced here and tested whether it could be used to denote the statistical characteristics of pedestrian movements.

Local density is a common concept used in crowd research studies, which describes the spatial occupancy of a small area around the pedestrian $[4,22,41]$. In a crowd, global density usually can successfully demonstrate the aggregation of pedestrians in the whole room or corridor, but the movements of a particular pedestrian are more likely to be affected by those pedestrians who are closer to him/her. In fact, if we go back to Figure 1, we can find that, in the original LGM, a pedestrian's movement in the next step is determined by the nearest three lattices, and this can be seen as a simplified version of the local density concept. Here, we adopted the method proposed by Helbing et al. [22] for calculation. Local density at a particular place $\vec{r}=(x, y)$ and time $t$ can be calculated by

$$
\rho(\vec{r}, t)=\frac{1}{S} \sum_{j} \exp \frac{-\left\|\vec{r}_{j}(t)-\vec{r}\right\|^{2}}{R^{2}},
$$

where $S$ is the surrounding area that local density calculation method would cover and $\vec{r}_{j}(t)$ is the position of pedestrian $j$ who is inside the surrounding area of $\vec{r}$. In this study, we assumed that a pedestrian will consider all others within his/ her visual field, so $S$ is denoted by the front visual area. Usually $S$ is a semicircle with the radius of measurement parameter $R$; thus, the area of $S$ is $0.5 \pi R^{2}$. However, if a pedestrian is close to the boundary, his/her front visual area will penetrate the wall of corridor, and $S$ should be a smaller value. The calculation method used here ignores the part of the surrounding area that crosses the boundary. Specifically, in Figure 6, two yellow areas show the method to determine the surrounding area. The left one indicates a pedestrian who is located at the middle part of the corridor while the right one shows a pedestrian who is near the boundary. The left one's surrounding area is the whole semicircle, and the left one's surrounding area should be the semicircle within the corridor.

2.3. Relationship between Local Density and Length of Movements. We used the above method to calculate the local density $\rho_{l}$ for each pedestrian at each step and obtain the moving distance in vertical $(x)$ and lateral $(y)$ direction in this step. Since the original dataset contains 6 experiments, we got 6 independent subdatasets whose format are all $\left[\rho_{l}, x\right.$, $y$ ]. In each experiment, the number of participants and the duration of the experiment vary from each other, so the size of each subdataset is different. In order to avoid statistical error caused by one particular subdataset, we randomly selected 500 items of data from each subdataset to create a new dataset.

In the new dataset, it is observed that $\rho_{l}$ ranges from 0 to $2.2 \mathrm{ped} / \mathrm{m}^{2}$. At a given local density, the length of pedestrian movements in each direction has some randomness, but from the statistical view, they may follow a certain distribution. So, according to $\rho_{l}$ the combined dataset is divided into 11 groups with interval of $0.2 \mathrm{ped} / \mathrm{m}^{2}$, which means that the $i$ th group contains the data with $\rho_{l}$ range in $[0.2 \cdot(i-1)$, $0.2 \cdot i)$. In each group, we assumed that the length of movements obey a distribution which is corresponding to the average local density of this group. Figures $7(a)$ and 7(c) show that the frequency distribution histograms of these movements could be fitted with a bell-curve, and in order to make the model simple and practical, normal distribution is employed to denote the dispersion of movements. The probability density function of normal distribution and parameters used here are shown below:

$$
f(x)=\frac{1}{\sqrt{2 \pi \beta^{2}}} \exp \left(-\frac{(x-\mu)^{2}}{2 \beta^{2}}\right),
$$

where $\mu$, location parameter, represents the average moving distance in a step and $\beta$ : scale parameter, represents the discreteness of moving distance in a step.

Maximum likelihood estimation (MLE) method is a dominant way for finding the best parameters of an assumed distribution which can fit the observed data better. In the following part, MLE is applied to figure out the location and scale parameter of each group of movements.

Figures 7(a) and 7(b) show the frequency of movements distance and the fitted parameters in $X$ direction, respectively. In Figure $7(\mathrm{a})$, we choose the two groups of data which the average $\rho_{l}$ equals 0.09 and $1.50 \mathrm{ped} / \mathrm{m}^{2}$ to demonstrate the distribution of movements distance at different local densities. It can be found that compared to low local density condition, the length of movements at high local density condition is closer to the origin point. In terms of the 


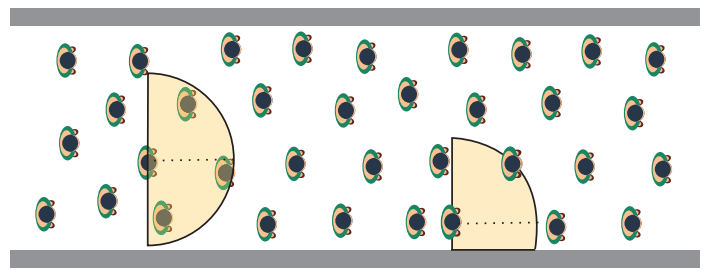

FIGURE 6: Sketch map of the front visual area.

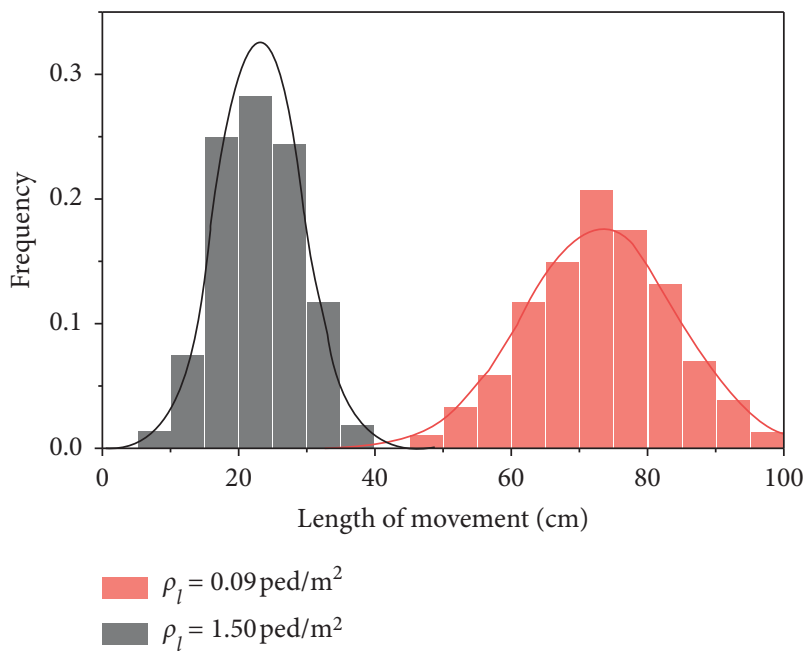

(a)

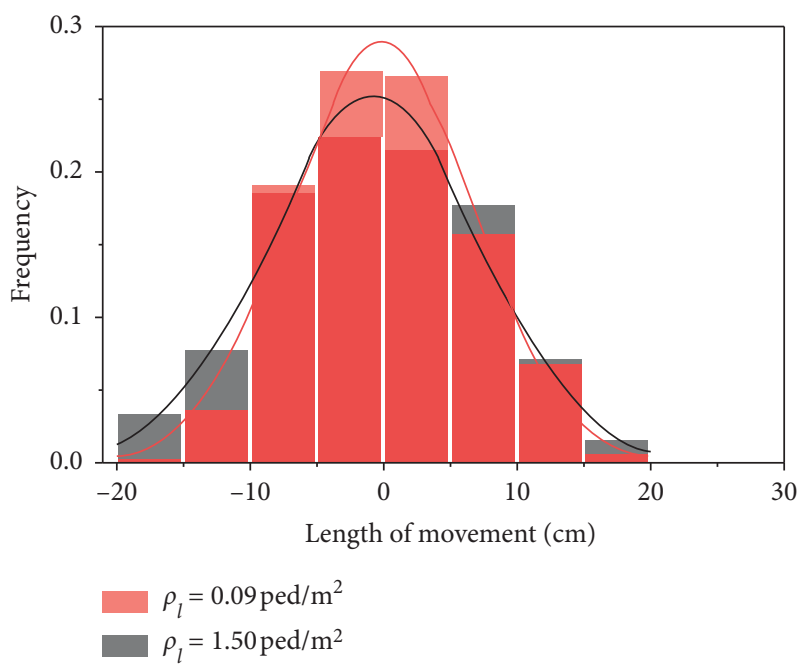

(c)

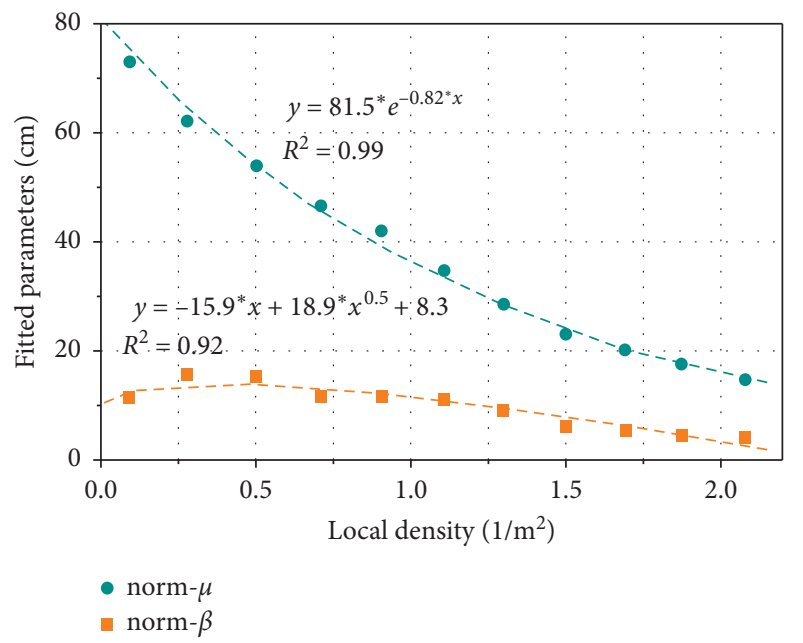

(b)

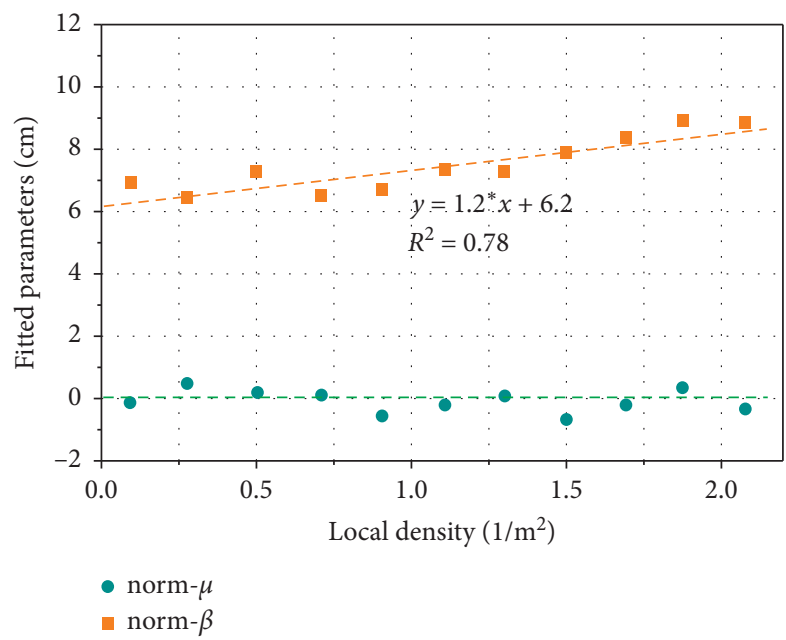

(d)

FIGURE 7: Fitted results of movements. (a) Movements in $X$ direction. (b) Fitted parameters of $X$ direction. (c) Movements in $Y$ direction. (d) Fitted parameters of $Y$ direction.

discreteness of movements, the red histograms are more dispersive than grey histograms, which implies that pedestrian movements in $\rho_{l}=1.50 \mathrm{ped} / \mathrm{m}^{2}$ are more short and centralized than movements in $\rho_{l}=0.09 \mathrm{ped} / \mathrm{m}^{2}$.

Figure 7(b) shows the fitted parameters at different local densities. The olive dots are the location parameters and orange dots are scale parameters. Fitted location parameters decline with the increase of local density, which indicates that pedestrian's moving strength is limited by the crowded surrounding environment. The R-square shows that exponential regression is suitable for denoting the relationship between location parameters and local density, and this also indicates that there is a good response relationship between them. The fitted scale parameters rise first and then decrease with the rise of local density, which shows that when the surrounding area is relatively empty, the pedestrian step in $X$ 
direction will centre in their maximum step size. And as the local density increase, the movements of pedestrian will become more discrete. When the local density reaches a relatively high state, the scale parameters decrease obviously, which can be a result of that the pedestrian's step size is all forced to keep in a limited small section. Therefore, we use a polynomial-like function to fit this first-rise-and-then-fall parameter.

In $X$ direction, pedestrian movements can be seen as subjecting to a normal distribution with the following parameters:

$$
\begin{aligned}
\mu & =81.5 \cdot \exp \left(-0.82 \cdot \rho_{l}\right), \\
R^{2} & =0.99, \\
\beta & =-15.9 \cdot \rho_{l}+18.9 \cdot \rho_{l}^{0.5}+8.3, \\
R^{2} & =0.92 .
\end{aligned}
$$

Figure 7 (c) shows the distribution of movements in $Y$ direction when the average $\rho_{l}$ equals 0.09 and $1.50 \mathrm{ped} / \mathrm{m}^{2}$. Both the red histograms and grey histograms can be regarded as symmetric distributed about the axis $x=0$, which indicates that the pedestrians have no preference to move to the left or right in this direction. In the aspect of discreteness, the red histograms are slightly more aggregated than the grey histograms, which show that the lateral dispersion effect in this direction is more obvious in higher local density condition.

Figure $7(d)$ is the fitted location and scale parameters in $Y$ direction. It shows that the mean length of movements always fluctuates around 0 , which is in accordance with what we observed in the videos that pedestrian has no lateral tendency. And the scale parameters have a slightly linear increase with the rising of local density, which means that the rising of local density will lead to a heavier vibration on pedestrian's lateral movements.

In $Y$ direction, pedestrian movements can be seen as subjecting to a normal distribution with the following parameters:

$$
\begin{aligned}
\mu & =0, \\
\beta & =1.2 \cdot \rho_{l}+6.2, \\
R^{2} & =0.78 .
\end{aligned}
$$

\section{Model Formulation}

Compared to the original LGM, the proposed model incorporates the statistical method to determine unidirectional crowd motion. The main modelling concept is that the pedestrian movements will follow some statistical rules. Our proposed model consists of two stages. The upper stage is the perceptional stage, where a pedestrian will calculate the local density of current position and receive two distributions. The lower stage is the operational stage, where a pedestrian will choose next step position based on other pedestrians' position, the boundary of corridor, and the confirmed distributions.
In the perceptional stage, pedestrians will scan the surrounding area and then apply the method in Section 2.2 to get the local density $\rho_{l}$ of current position. Then, accompanied with functions of local density and distribution' parameters (equations (3)-(6)), the pedestrian will have two PDFs that describe the movements in $X$ and $Y$ direction.

In the operational stage, the model's main task is to generate a vector $l=\left(l_{x}, l_{y}\right)$ for the pedestrian to move. The $l_{x}$ and $l_{y}$ are random variates which follow the confirmed distribution in $X$ and $Y$ direction, respectively. Yet, in this process, if the pedestrian directly adopts the random vector $l$, he/she may face a problem of collision, which is usually not acceptable in real scenarios. In the model, there are two types of collision, one is overlapping with other pedestrians and the other is crossing the boundary. As shown in Figure 8, the red circle represents the subject pedestrian and blue circles represent surrounding pedestrians. The grey rectangle indicates the wall. After the model generates a vector, the pedestrian is going to move by this vector. If the new position will overlap with others or cross the wall, as shown in Figure 8 by dash arrows and circles, the model will abandon this vector and regenerate new random vector. If the generated vector will not lead to any type of collision, the pedestrian will adopt this vector.

To solve the problem of collision, we introduced a loop in this stage. The model will first enter a loop, which continues to generate random vectors until one of the following two conditions is met. One is that the generated vector will not lead to collision, and the other is that the number of times to regenerate the vector reaches acceptable trying times (ATT). Here, the acceptable trying times determine how many times the model will generate the vector for a pedestrian in a step. In the original trajectories, we found that even at a very high density, the pedestrian will still move by a short length, rather than keep stationary; or to say, the frequency of step $=(0,0)$ is almost nonexistent. We have to conduct some simulation and it shows that if the ATT is set as a small value such as 10 or 100 , the pedestrians will frequently keep stationary, and if the value is increased to 1000, the model will have a much better performance. If the value continues to increase to 10000 or large, the model will consume lots of computation power with little considerable improvement. So, in the model, we set the ATT as a relatively big value as 1000 .

Here, the model employs random sequential update rule to renew pedestrian's position. Specifically, pedestrian's position is updated one by one in a given order at each time step, and this order is generated randomly at every step.

The updating of a pedestrian's position can be realized by the following steps:

Step 1: obtain local density. Scan all pedestrians within the surrounding area and calculate the local density $\rho_{l}$.

Step 2: determine the PDF of movement in each direction. Use $\rho_{l}$ and equations (3)-(6) to derive the parameters of distribution in $X$ and $Y$ direction, and return corresponding $\mathrm{PDF}$. 


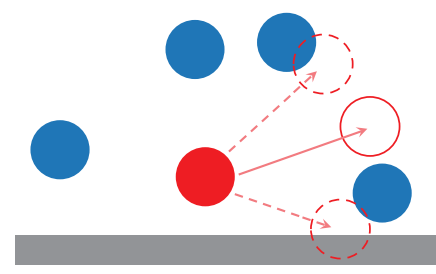

Figure 8: Pedestrian's collision avoidance.

Step 3: generate the moving distance.

$$
\text { For } i=1 ; i \leq \mathrm{ATT} ; i++
$$$$
\{
$$

Generate a vector $l=\left(l_{x}, l_{y}\right)$. Here $l_{x}$ and $l_{y}$ are random variate which follows the distribution of $X$ direction and $Y$ direction, respectively.

If $l$ not collides with other pedestrians or crossing the boundary:

Return $l=\left(l_{x}, l_{y}\right)$, and quit the loop;

Else:

$$
\begin{aligned}
& \text { Abandon the vector } l \text {. } \\
& \text { If } i>\text { ATT: } \\
& \text { Return } l=(0,0)
\end{aligned}
$$

Step 4: update pedestrian's position. The pedestrian moves along the determined vector $l$.

The flow chart below shows the entire procedure (Figure9).

\section{Simulation and Results}

We conducted a series of simulation using the scenario shown in Figure 5 to validate the model. The length and width of the corridor is $16 \mathrm{~m}$ and $5 \mathrm{~m}$, respectively. The amount of pedestrians in the model is changed from 5 to 250 by step of 5 to simulate different global densities. In the simulation, pedestrians were randomly placed in the corridor before traversing the corridor. Close boundary condition is used here to keep the number of pedestrians in the model unchanged and the global density constant. In this model, pedestrians are denoted by a circle with a radius of $0.2 \mathrm{~m}$, and the time step is set as $0.5 \mathrm{~s}$, which is the same as in the previous analysis. The duration of each simulation is 500 steps, equals to $250 \mathrm{~s}$.

Figure 10 is a typical snapshot of the simulation. The colorful circles represent pedestrians. From the snapshots, it can be found that the pedestrian positions are relatively disordered. Compared with the original LGM that all pedestrians have to keep in align, the proposed model is closer to reality. The disordered positions of crowd can be seen as a result of continuous step length and unlimited heading directions employed in the proposed model. In order to be consistent with the experimental setup, the trajectories within the canter part of the corridor are used for subsequent analysis, which is denoted in Figure 10 by the blue square.

The fundamental diagrams are employed to show the numerical results of the model. In Figure 11(a), the colorful

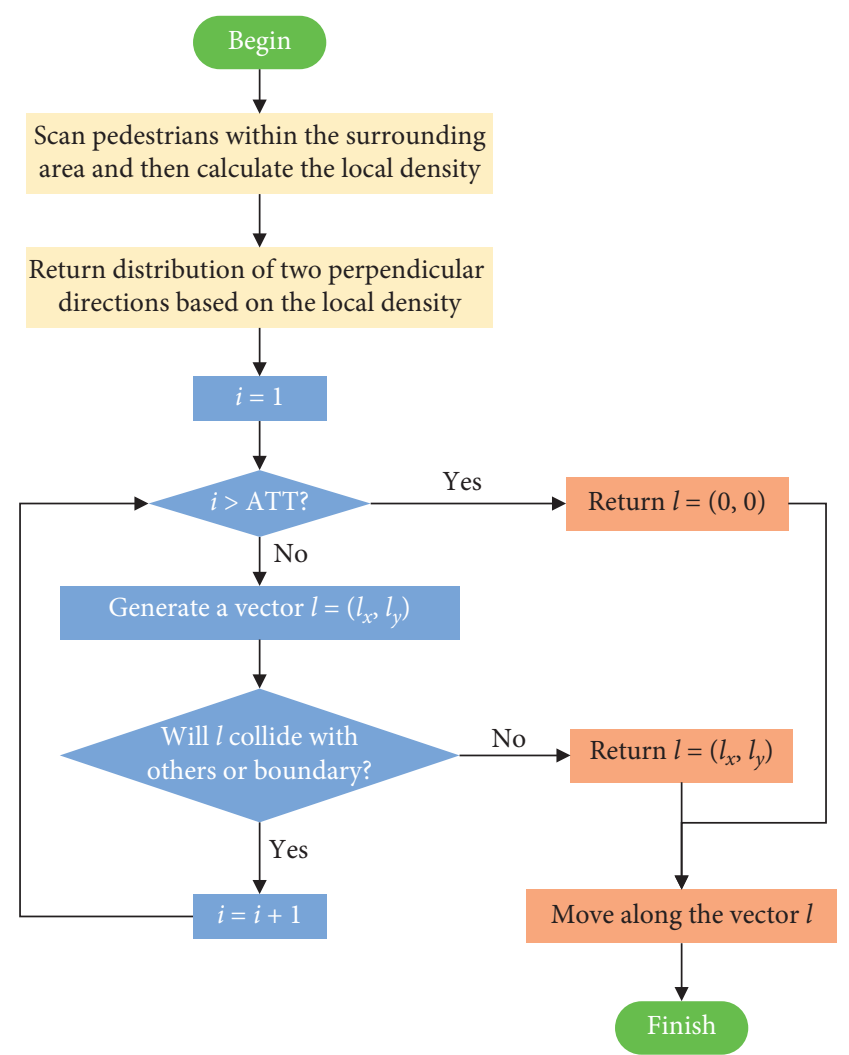

FIgURE 9: Flow chart of the updating procedure.

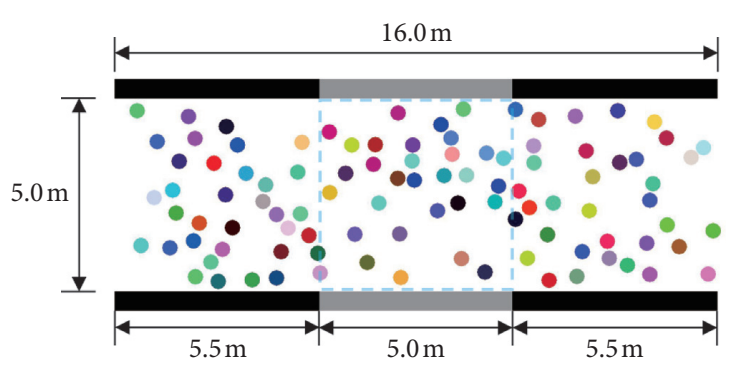

FIgURE 10: Snapshot of 100 pedestrians at 200th step.

square dots represent speed-density pairs of experimental data from the realistic trajectories, the grey circle dots are the simulated speed-density pairs from the proposed model, and the four curves represent the speed-density relationships derived from the original LGM with a different drift strength $D$ and time step $T$. It can be found that the experimental data are usually more dispersed than those from simulation, and the simulated speed-density dots from proposed model cross nearly the middle of the experimental dots. Here, based on the level of global density, we determine three typical conditions. Condition A represents the low global density situation with $0.2-0.4$ pedestrians per square. Condition B represents the medium situation with global density among 1.6-1.8 ped $/ \mathrm{m}^{2}$ and condition $\mathrm{C}$ stands for the high global density condition where the density is among 2.8-3.0 ped $/ \mathrm{m}^{2}$. We chose four typical curves from the original LGM to illustrate the simulation effect. From the figure, it shows the speed-density relationships of the original 


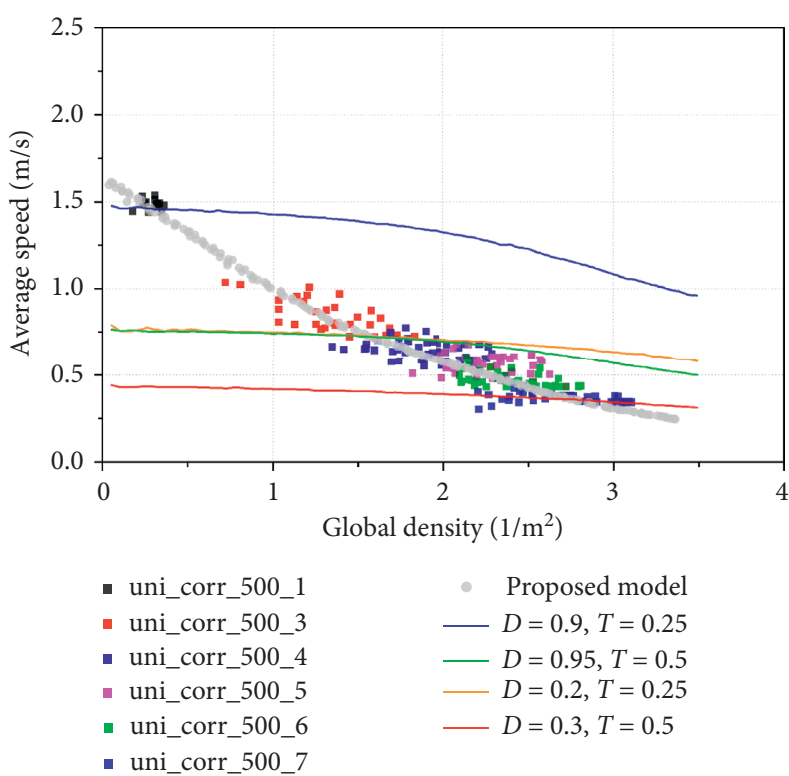

(a)

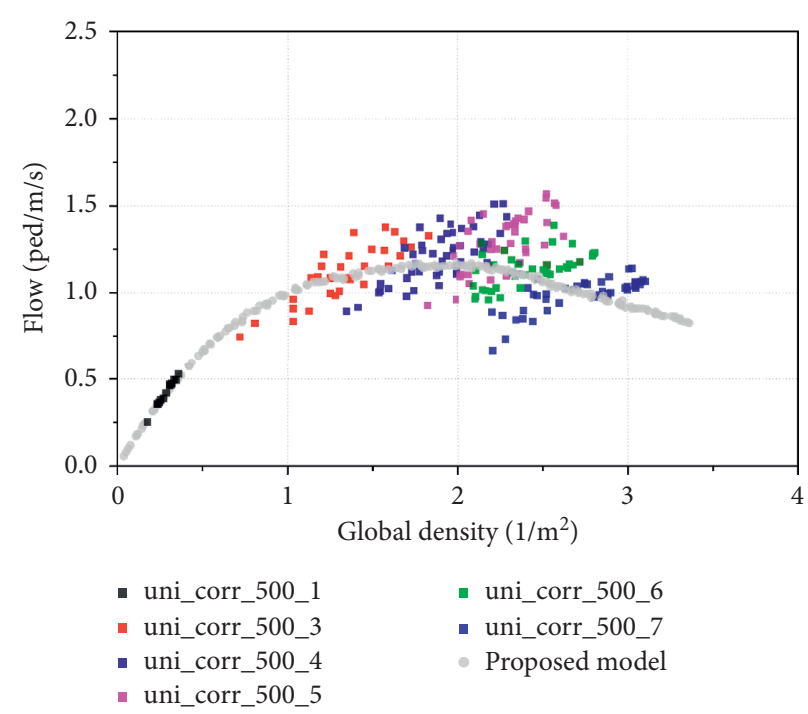

(b)

FIgURE 11: Fundamental diagrams. (a) Speed-density diagram. (b) Flow-density diagram.

LGM with $D=0.9$, and $T=0.25 \mathrm{~s}$ can reflect the pedestrian behaviour at low global density condition but fails to fit it at conditions $\mathrm{B}$ and $\mathrm{C}$, and if $D$ is declined to 0.2 or $D=0.95$ and $T=0.5 \mathrm{~s}$ are used, the fitting result in condition $\mathrm{B}$ is acceptable but there is an obvious deviation in low and high global density condition. Speed-density relationships at $D=0.3$ and $T=0.5 \mathrm{~s}$ can reproduce it at condition $\mathrm{C}$ but is not sensitive at conditions $\mathrm{A}$ and $\mathrm{B}$. Therefore, we can believe that the proposed model can promote the fitness of speed-density relationships of unidirectional pedestrian movements in the LGM.

Figure 11(b)is flow-density diagram. Since the flowdensity pairs of original LGM vary hugely with the experimental data, we did not show it in the figure. At the low global density condition of less than 2 pedestrians per square, the flow increases with the rising of density and reaches $1.2 \mathrm{ped} / \mathrm{s} / \mathrm{m}$. Then, the flow declines with the growth of density. Compared to the flow-density pairs from experimental data, the simulated pairs are more concentrated. This is because that in realistic walking process all pedestrians are not totally homogeneous and have some stochastic behaviours, but in the LGM all particles are set to obey one identical rule. Even though, from the similarity of experimental and simulated data in fundamental diagrams, we can believe that this model can reappear the general speed-density and flowdensity relationship of realistic pedestrian trajectory.

We also investigated the transition probability of pedestrian at different global densities. In the model, local density is used to determine pedestrian movements, while in most application scenarios, the engineers or scientists care more about the global density. The potential movement area is discrete into $80001 \mathrm{~cm} * 1 \mathrm{~cm}$ girds to show the transition probability of different points. Figure 12 shows transition probability derived from realistic pedestrian trajectories and simulation at different global densities. Here, the three typical conditions mentioned above are used to analyse the transition probability. Figure 12 (a) is the transition probability of realistic pedestrian trajectories at condition $\mathrm{A}$ and Figure 12(b) is the result from simulation. In this condition, the interquartile range (IQR) of realistic pedestrian movements is $x \in[-4.8,4.2]$ and $y \in[61,75.7]$, and the IQR of simulation is $x \in[-5.1,5.1]$ and $\mathrm{y} \in[62.9$. 79.6]. In condition $\mathrm{B}$, the IQR of realistic trajectory is $x \in[-5.5,4.8]$ and $y \in[27.4,43.9]$ (Figure 12(c)), and the IQR of simulated steps is $x \in[-5.1,5.0]$ and $\mathrm{y} \in[28.3,45.8]$ (Figure 12(d)). As the global density increased to condition $\mathrm{C}$, the IQR of realistic trajectory is $x \in[-5.9,6.0]$ and $y \in[13.8,21.2]$ (Figure 12(c)), and the IQR of simulated steps is $x \in[-5.5,5.3]$ and $y \in[11.7$, 19.3] (Figure 12(d)). Because we used some approximate calculation and regression method in parameter analysis, there is a little deviation between transition probability of realistic trajectories and simulation. However, compared to the previous models with discontinuous step size, the proposed model can describe transition probability at any possible position, rather than limited neat points.

The Generalized Euclidean Distance (GED) can be used to evaluate the fitting result. GED between two groups of transition probability is

$$
\mathrm{GED}=\sqrt{\sum_{i}\left(p_{1, i}-p_{2, i}\right)^{2}}
$$

where $p_{1, i}$ is the transition probability of $i$ th grid in group 1 and $p_{2, i}$ is the transition probability of $i$ th grid in group 2;

The GED of transition probability between realistic and simulation is 0.042 in condition $\mathrm{A}, 0.017$ in condition $\mathrm{B}$, and 0.031 in condition C. When original LGM with $D=0.9$ and $T=0.25 \mathrm{~s}$ is employed for condition A, the GEM is 0.870 , and for condition B, the GEM is 0.456 (when $D=0.2$ and $T=0.25 \mathrm{~s}$ ) and 0.909 (when $D=0.95$ and $T=0.5 \mathrm{~s}$ ). In condition C, the GEM is 0.554 when $D=0.3$ and $T=0.5 \mathrm{~s}$. 


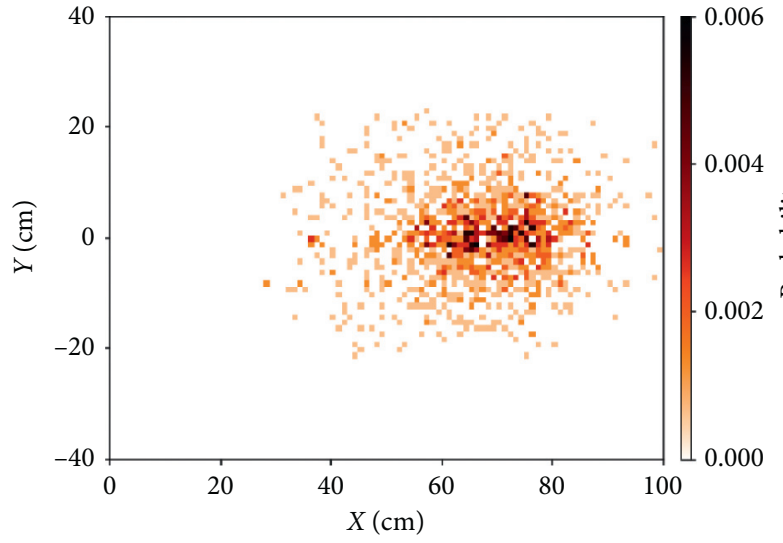

(a)

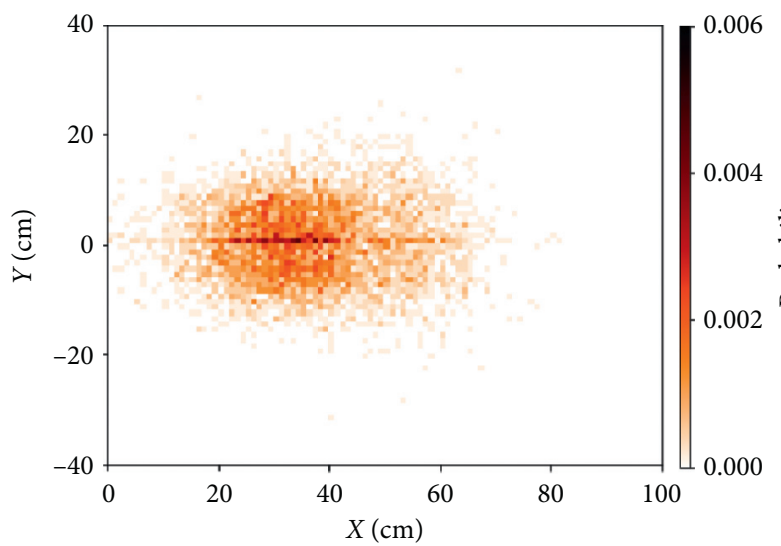

(c)

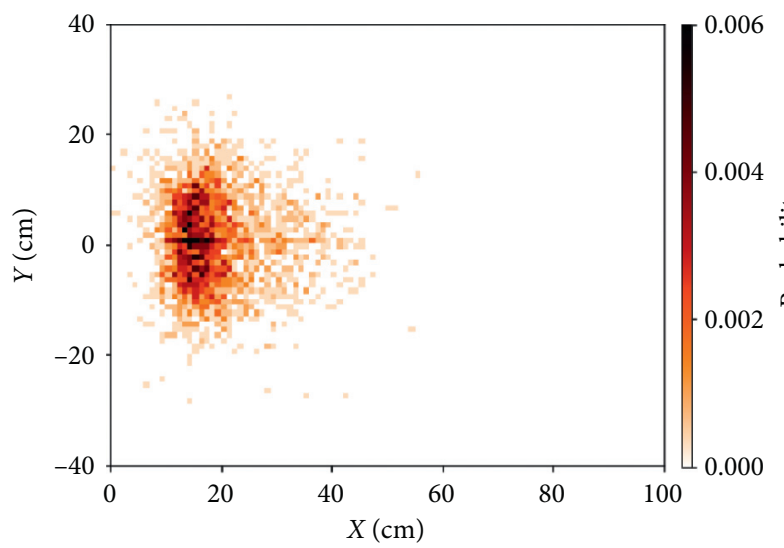

(e)

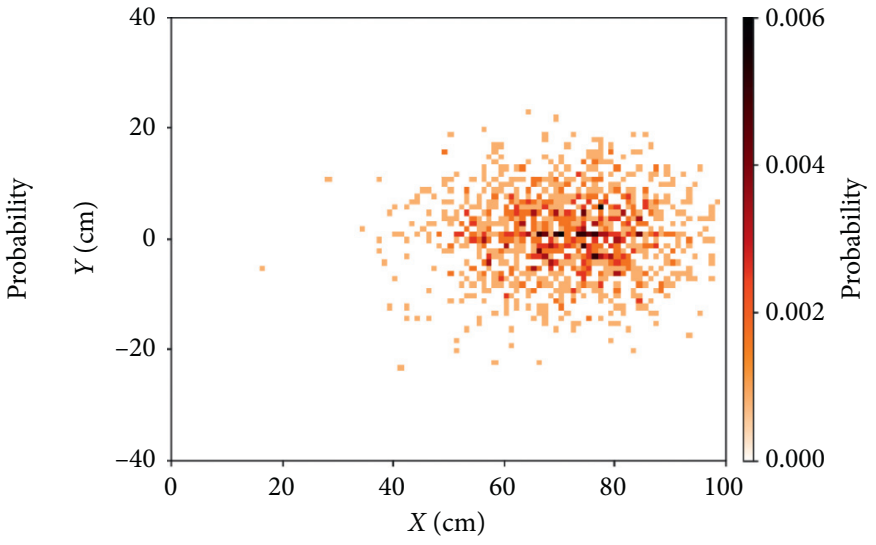

(b)

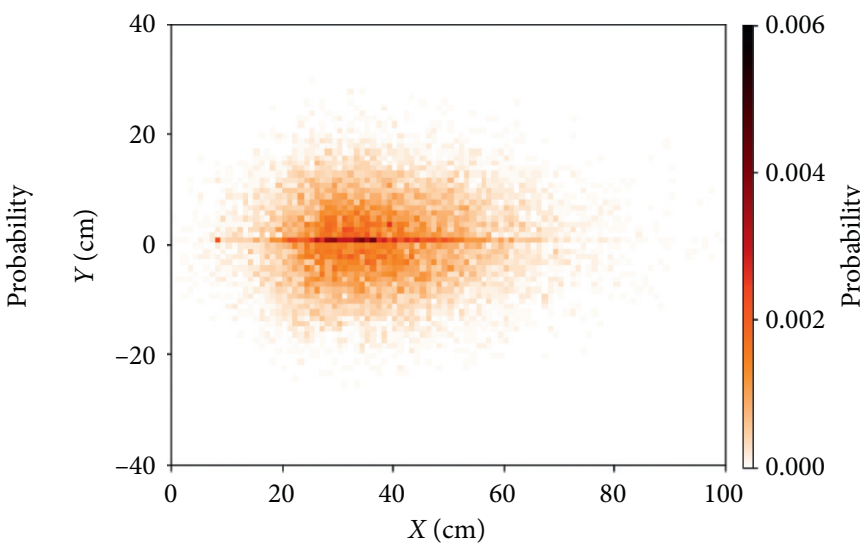

(d)

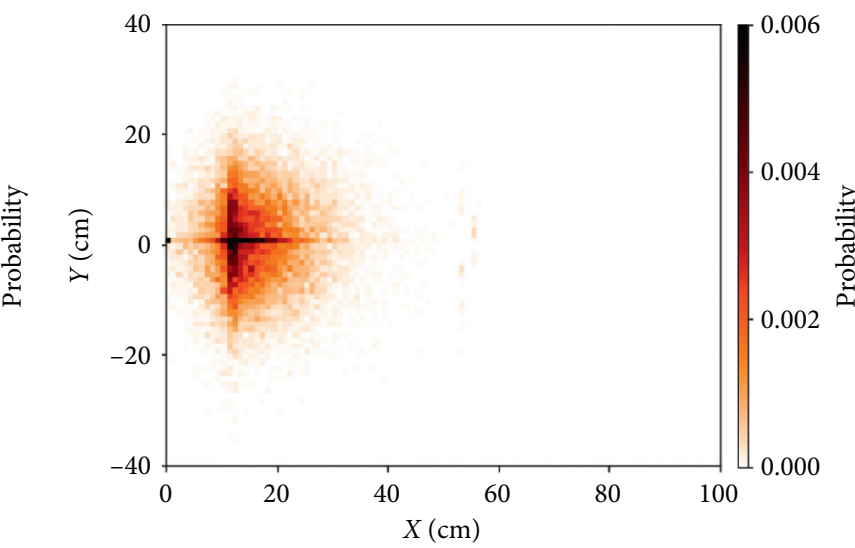

(f)

FIgURE 12: Transition probabilities. (a) Global density $=[0.2,0.4]$, realistic. (b) Global density $=[0.2,0.4]$, simulated. (c) Global density $=$ $[1.6,1.8]$, realistic. (d) Global density $=[1.6,1.8]$, simulated. (e) Global density $=[2.8,3.0]$, realistic. (f) Global density $=[2.8,3.0]$, simulated.

The higher GED in the original LGM is because there are just several finite potential points for pedestrian to move in this model, yet the relatively low GEM in the proposed model implies the good applicability of our model.

\section{Conclusions}

In this study, we proposed an extended LGM for unidirectional pedestrian flow in a corridor. Our work is motivated by the statistical characteristics of pedestrian movements and the unreasonable transition probability caused by discontinuous step length and finite heading directions in the original LGM.

We used an open dataset to derive the relationship between pedestrian movements and local density and then incorporated these rules into the original LGM for determining pedestrian's position. By the maximum likelihood estimation method, we found normal distribution with fitted 
parameters to denote pedestrian's lateral and vertical movements. The parameters of these distributions can also be regarded as varying reasonably with local density. The model is applied to a scenario which is the same as the experimental setup, and the speed-density and flow-density relationship obtained from the simulations are consistent with that derived from the experimental trajectories. Besides, the transition probability to each position at different global densities in realistic trajectories and simulation shows that the model can successfully reproduce the statistical characteristics of pedestrian movements.

With the proposed extended LGM, we can simulate pedestrian movements without fixed step size and direction and provide pedestrian with a more accurate transition probability. However, this model just considered the statistical characteristics of pedestrian's two-dimensional movements in a general condition. The parameters acquired in this study are based on the data from BaSiGo, which focuses on the corridor and the participants are majorly students. For other scenarios, such as stairs or sidewalks, the method shown in this study can be applied to calibrate parameters. In fact, numerous studies have found that many factors will influence pedestrian's behaviour, such as group effect [2,31], age or gender composition [42], and handicapped people [19]. And in bidirectional crowd motion, pedestrian lateral movements are not as random as unidirectional movements because opposite pedestrians will have an oppressive effect on their lateral movements. Future research studies can further study these factors' influence on the statistical characteristics of pedestrian movements and incorporate these to make the model more accurate and universal.

\section{Data Availability}

The data used to support the findings of this study are available from the corresponding author upon request.

\section{Conflicts of Interest}

The authors declare that they have no conflicts of interest.

\section{Acknowledgments}

This research was supported by the key project of National Natural Science Foundation of China (nos. 51878166, 71801042, and 71701047), Postgraduate Research and Practice Innovation Program of Jiangsu Province (no. SJCX18_0042), and Natural Science of Jiangsu Province (no. BK20180381).

\section{References}

[1] W. Song, X. Xu, B.-H. Wang, and S. Ni, "Simulation of evacuation processes using a multi-grid model for pedestrian dynamics," Physica A: Statistical Mechanics and Its Applications, vol. 363, no. 2, pp. 492-500, 2006.

[2] L. Lu, C.-Y. Chan, J. Wang, and W. Wang, "A study of pedestrian group behaviors in crowd evacuation based on an extended floor field cellular automaton model,"
Transportation Research Part C: Emerging Technologies, vol. 81, pp. 317-329, 2017.

[3] W. Zeng, P. Chen, H. Nakamura, and M. Iryo-Asano, "Application of social force model to pedestrian behavior analysis at signalized crosswalk," Transportation Research Part C: Emerging Technologies, vol. 40, pp. 143-159, 2014.

[4] G. Ren, L. Lu, W. Wang, X. Gong, and Z. Huang, "Microscopic simulation model for pedestrian flow at signalized crosswalks," Transportation Research Record: Journal of the Transportation Research Board, vol. 2434, no. 1, pp. 113-122, 2014.

[5] H.-Y. Shang, H.-J. Huang, and Y.-M. Zhang, "An extended mobile lattice gas model allowing pedestrian step size variable," Physica A: Statistical Mechanics and Its Applications, vol. 424, pp. 283-293, 2015.

[6] G. Qiu, R. Song, S. He, and W. Yin, "The pedestrian flow characteristics of Y-shaped channel," Physica A: Statistical Mechanics and Its Applications, vol. 508, pp. 199-212, 2018.

[7] D. Helbing and P. Molnár, "Social force model for pedestrian dynamics," Physical Review E, vol. 51, no. 5, pp. 4282-4286, 1995.

[8] M. Muramatsu, T. Irie, and T. Nagatani, "Jamming transition in pedestrian counter flow," Physica A: Statistical Mechanics and Its Applications, vol. 267, no. 3-4, pp. 487-498, 1999.

[9] V. J. Blue and J. L. Adler, "Cellular automata microsimulation of bidirectional pedestrian flows," Transportation Research Record: Journal of the Transportation Research Board, vol. 1678, no. 1, pp. 135-141, 1999.

[10] A. Kirchner and A. Schadschneider, "Simulation of evacuation processes using a bionics-inspired cellular automaton model for pedestrian dynamics," Physica A: Statistical Mechanics and Its Applications, vol. 312, no. 1-2, pp. 260-276, 2002.

[11] Krockenberger, M. S Asselov, and D. D. N Oyes, "Simulating dynamical features of escape panic," Nature, vol. 144, pp. 297-311, 2000.

[12] R. Liu, Z. Fu, A. Schadschneider, Q. Wen, J. Chen, and S. Liu, "Modeling the effect of visibility on upstairs crowd evacuation by a stochastic FFCA model with finer discretization," Physica A: Statistical Mechanics and Its Applications, vol. 531, Article ID 121723, 2019.

[13] R. Ye, M. Chraibi, C. Liu et al., "Experimental study of pedestrian flow through right-angled corridor: uni- and bidirectional scenarios," Journal of Statistical Mechanics: Theory and Experiment, vol. 2019, no. 4, Article ID 043401, 2019.

[14] Z. Shahhoseini and M. Sarvi, "Pedestrian crowd flows in shared spaces: investigating the impact of geometry based on micro and macro scale measures," Transportation Research Part B: Methodological, vol. 122, pp. 57-87, 2019.

[15] S. Cao, A. Seyfried, J. Zhang, S. Holl, and W. Song, "Fundamental diagrams for multidirectional pedestrian flows," Journal of Statistical Mechanics: Theory and Experiment, vol. 2017, no. 3, Article ID 033404, 2017.

[16] G. Zeng, A. Schadschneider, J. Zhang, S. Wei, W. Song, and R. Ba, "Experimental study on the effect of background music on pedestrian movement at high density," Physics Letters A, vol. 383, no. 10, pp. 1011-1018, 2019.

[17] S. Cao, P. Wang, M. Yao, and W. Song, "Dynamic analysis of pedestrian movement in single-file experiment under limited visibility," Communications in Nonlinear Science and Numerical Simulation, vol. 69, pp. 329-342, 2019.

[18] P. Geoerg, J. Schumann, S. Holl, and A. Hofmann, "The influence of wheelchair users on movement in a bottleneck and a corridor," Journal of Advanced Transportation, vol. 2019, Article ID 9717208, 17 pages, 2019. 
[19] J. Kim, C. Ahn, and S. Lee, "Modeling handicapped pedestrians considering physical characteristics using cellular automaton," Physica A: Statistical Mechanics and Its Applications, vol. 510, pp. 507-517, 2018.

[20] C. Chen, Y. Tong, C. Shi, and W. Qin, "An extended model for describing pedestrian evacuation under the threat of artificial attack," Physics Letters A, vol. 382, no. 35, pp. 2445-2454, 2018.

[21] J. Zhang, W. Klingsch, A. Schadschneider, and A. Seyfried, "Transitions in pedestrian fundamental diagrams of straight corridors and T-junctions," Journal of Statistical Mechanics: Theory and Experiment, vol. 2011, no. 6, Article ID P06004, 2011.

[22] D. Helbing, A. Johansson, and H. Z. Al-Abideen, "Dynamics of crowd disasters: an empirical study," Physical Review E Statistical, Nonlinear, and Soft Matter Physics, vol. 75, no. 4, 2007.

[23] S. Holl, Methoden für die Bemessung der Leistungsfähigkeit multidirektional genutzter Fußverkehrsanlagen, Forschungszentrum Jülich GmbH Zentralbibliothek, Verlag, Zentralbibliothek, Jülich, Germany, 2016.

[24] J. Zhang, W. Klingsch, A. Schadschneider, and A. Seyfried, "Ordering in bidirectional pedestrian flows and its influence on the fundamental diagram," Journal of Statistical Mechanics: Theory and Experiment, vol. 2012, no. 2, Article ID P02002, 2012.

[25] A. Sieben, J. Schumann, and A. Seyfried, "Collective phenomena in crowds-Where pedestrian dynamics need social psychology," PLoS One, vol. 12, no. 6, pp. 1-19, 2017.

[26] M. Chraibi, A. Seyfried, and A. Schadschneider, "Generalized centrifugal-force model for pedestrian dynamics," Physical Review E - Statistical, Nonlinear, and Soft Matter Physics, vol. 82, no. 4, pp. 1-7, 2010.

[27] V. J. Blue and J. L. Adler, "Cellular automata microsimulation for modeling bi-directional pedestrian walkways," Transportation Research Part B: Methodological, vol. 35, no. 3, pp. 293-312, 2001.

[28] J. Liang, Y. Zhang, and J. Yang, "An extended small-grid lattice gas model for pedestrian counter flow," Procedia Engineering, vol. 62, pp. 501-508, 2013.

[29] X. Song, J. Sun, H. Xie, Q. Li, Z. Wang, and D. Han, "Characteristic time based social force model improvement and exit assignment strategy for pedestrian evacuation," Physica A: Statistical Mechanics and Its Applications, vol. 505, pp. 530-548, 2018.

[30] B. Ni, Z. Lin, and P. Li, "Agent-based evacuation model incorporating life jacket retrieval and counterflow avoidance behavior for passenger ships," Journal of Statistical Mechanics: Theory and Experiment, vol. 2018, no. 12, Article ID 123405, 2018.

[31] V. J. Blue and J. L. Adler, "Emergent fundamental pedestrian flows from cellular automata microsimulation," Transportation Research Record: Journal of the Transportation Research Board, vol. 1644, no. 1, pp. 29-36, 1998.

[32] R.-Y. Guo, "New insights into discretization effects in cellular automata models for pedestrian evacuation," Physica A: Statistical Mechanics and Its Applications, vol. 400, pp. 1-11, 2014.

[33] J. Ji, L. Lu, Z. Jin, S. Wei, and L. Ni, "A cellular automata model for high-density crowd evacuation using triangle grids," Physica A: Statistical Mechanics and Its Applications, vol. 509, pp. 1034-1045, 2018.

[34] L. Tu and J. Zhou, "Memory's effect on bidirectional pedestrian flow based on lattice hydrodynamic model," Physica
A: Statistical Mechanics and Its Applications, vol. 534, Article ID 122225, 2019.

[35] D. Miyagawa and G. Ichinose, "Cellular automaton model with turning behavior in crowd evacuation," Physica A: Statistical Mechanics and Its Applications, vol. 549, Article ID 124376, 2020.

[36] Z.-J. Ding, Y.-H. Huang, T. Liu et al., "Analytical and simulation studies of intersecting pedestrian flow on the $2 \mathrm{D}$ lattice with parallel update rule," Physica A: Statistical Mechanics and Its Applications, vol. 523, pp. 1183-1201, 2019.

[37] R. Y. Guo and H. J. Huang, "A mobile lattice gas model for simulating pedestrian evacuation," Physica A: Statistical Mechanics and Its Applications, vol. 387, no. 2-3, pp. 580-586, 2008.

[38] R.-Y. Guo, S. C. Wong, H.-J. Huang, P. Zhang, and W. H. K. Lam, "A microscopic pedestrian-simulation model and its application to intersecting flows," Physica A: Statistical Mechanics and Its Applications, vol. 389, no. 3, pp. 515-526, 2010.

[39] X. Song, D. Han, J. Sun, and Z. Zhang, "A data-driven neural network approach to simulate pedestrian movement," Physica A: Statistical Mechanics and Its Applications, vol. 509, pp. 827-844, 2018.

[40] S. Armin and B. Maik, "Pedestrian dynamics data archive," 2013, http://ped.fz-juelich.de/da/.

[41] F. Johansson, A. Peterson, and A. Tapani, "Waiting pedestrians in the social force model," Physica A: Statistical Mechanics and Its Applications, vol. 419, pp. 95-107, 2015.

[42] X. Ren, J. Zhang, W. Song, and S. Cao, "The fundamental diagrams of elderly pedestrian flow in straight corridors under different densities," Journal of Statistical Mechanics: Theory and Experiment, vol. 2019, no. 2, Article ID 023403, 2019. 